Meta

Journal des traducteurs

Translators' Journal

\title{
La formation à la recherche traductologique et le concept CERA Chair
}

\section{Daniel Gile}

Volume 41, numéro 3, septembre 1996

URI : https://id.erudit.org/iderudit/002888ar

DOI : https://doi.org/10.7202/002888ar

Aller au sommaire du numéro

Éditeur(s)

Les Presses de l'Université de Montréal

ISSN

0026-0452 (imprimé)

1492-1421 (numérique)

Découvrir la revue

Citer cette note

Gile, D. (1996). La formation à la recherche traductologique et le concept CERA

Chair. Meta, 41(3), 486-490. https://doi.org/10.7202/002888ar
Résumé de l'article

L'auteur expose un programme universitaire original pour les jeunes chercheurs traducto-logues: la CERA Chair for Translation, Communication and Cultures. Il présente quelques caractéristiques originales et intéressantes du programme et décrit le travail des stagiaires avec des données recueillies au cours de la session 1993. 


\section{BLOC-NOTES}

\section{LA FORMATION À LA RECHERCHE TRADUCTOLOGIQUE ET LE CONCEPT CERA CHAIR}

\section{Résumé}

L'auteur expose un programme universitaire original pour les jeunes chercheurs traductologues: la CERA Chair for Translation, Communication and Cultures. Il présente quelques caractéristiques originales et intéres santes du programme et décrit le travail des stagiaires avec des données recueillies au cours do la session 1993.

\section{Abstract \\ CERA (Chair for Translation, Communication and Cultures) is a unique university programme for new researchers in translatology. In this article, the author presents some of the special features of the programme and reports on research conducted during the 1993 session.}

La traductologie est une discipline jeune, qui n'a pas encore pignon sur rue dans le monde universitaire: si les écoles de traduction sont désormais nombreuses, les départements de traductologie au sens universitaire du terme (par opposition aux programmes de formation professionnelle) restent très rares, et la grande majorité des traductologues appartiennent à des départements de langues vivantes, de linguistique, de littérature. En corollaire, les traductologues ont du mal à trouver une formation spécifique, ce qui se traduit notamment par des faiblesses méthodologiques dans la recherche qui, nous semblet-il, pourraient être évitées (voir Toury 1991 et Gile 1991).

Sur cette toile de fond se dessine un programme original, dont l'initiateur est José Lambert, de la Katholieke Universiteit Leuven, en Belgique: le stage de perfectionnement à la recherche traductologique de la CERA Chair for Translation, Communication and Cultures. Il s'agit d'un programme annuel d'un mois de travail individuel et collectif à Louvain, pendant lequel sont accueillis et orientés quelque 20 jeunes chercheurs traductologues. Pour les 15 premiers jours, le programme prévoit des conférences et séminaires, qui sont réalisés par une équipe d'animateursdirecteurs de recherche, tous chercheurs confirmés, ainsi que par le professeur CERA, nommé annuellement. La deuxième quinzaine est essentiellement consacrée au travail individuel des stagiaires, qui présentent tour à tour l'état d'avancement de leurs travaux. Tout au long de cette période, les animateursdirecteurs de recherche sont à la disposition des stagiaires pour des entretiens d'orientation individuels, qui s'ajoutent au programme collectif.

Le programme, subventionné par la banque CERA, a démarré en 1989. Les professeurs CERA nommés jusqu'à présent étaient Gideon Toury de TelAviv (1989), Hans Vermeer de Heidelberg (1990), Susan Bassnett de Warwick (1991), Albrecht Neubert de Leipzig (1992), et Daniel Gile de Paris (1993).

Si l'idée d'un stage de formation estival n'a rien de révolutionnaire en soi, le programme CERA a quelques caractéristiques originales et très intéressantes dans le domaine traductologique.

- Il s'agit d'un programme court et intensif axé sur la méthodologie générale de la recherche, par opposition à l'acquisition d'une théorie particulière ou d'une technique de recherche spécifique. Les cours de méthodologie de la recherche en tant que tels sont quasiment inexistants en traductologie.

- Le programme est résolument inter-universitaire et international. Les animateurs viennent de Belgique (Anvers, Louvain, Namur), mais aussi de Londres et de Göttingen, avec des intervenants extérieurs des Pays-Bas, des États-Unis, d'Israël, d'Allemagne et de France selon les années, la liste n'étant pas limitative. Quant aux stagiaires, ils viennent aussi de pays très variés : en 1993, les pays représentés étaient l'Allemagne, l'Australie, l'Autriche, la Belgique, le Danemark, l'Espagne, la Finlande, la Grèce, la Hongrie, l'Irlande, l'Italie, la Pologne, le Royaume-Uni; les années précédentes, des stagiaires africains et asiatiques figuraient également parmi les participants. La langue de travail officielle est l'anglais.

Ce caractère international du programme nous semble particulièrement important compte tenu du petit nombre de traductologues et d'apprentistraductologues dans le monde et de leur grande dispersion géographique. 
- Le rapport entre le nombre d'animateurs et le nombre de stagiaires est particulièrement intéressant: en 1993, une dizaine d'encadrants pour un peu plus de 20 stagiaires. Il est vrai qu'une partie d'entre eux ne sont restés que quelques jours, mais cing sont restés soit une semaine entière, soit deux à trois jours par semaine sur l'ensemble de la période, assurant ainsi une certaine continuité. Chaque stagiaire a donc eu la possibilité de s'entretenir individuellement de son projet de recherche avec une dizaine de chercheurs confirmés, dont certaines personnalités fort connues dans le monde traductologique. Ne serait-ce que sur cet aspect précis, le programme CERA est très précieux.

D'après les explications données dans les documents de présentation du programme, celui-ci se veut interdisciplinaire. En réalité, cette interdisciplinarité est plutôt limitée, dans la mesure où la plupart des animateurs-directeurs de recherche sont issus d'études littéraires et ont fait l'essentiel de leur travail de recherche initial dans le champ littéraire. Il règne toutefois dans l'equipe un esprit d'ouverture certain. En témoigne notamment le fait que l'équipe CERA, qui préconise la démarche «descriptive» (voir Delabastita 1992), désignée ainsi par référence oppositionnelle à la démarche normative qui caractérise un grand nombre de recherches traductologiques (voir Toury 1980), a nommé comme deuxième professeur CERA Hans Vermeer, dont la philosophie est très éloignée de la sienne. La même ouverture d'esprit a présidé à la nomination comme professeur CERA de D. Gile, interprète de conférence et traducteur scientifique et technique qui n'a pas de compétence littéraire. $\mathrm{Au}$ cours des séminaires et discussions auxquels nous avons assisté pendant la session 1993, nous avons constaté la même ouverture d'esprit.

Le travail des stagiaires commence par la lecture des articles qui leur sont envoyés quelques mois avant le début de la session à titre de préparation (plus de 100 pages de texte, choisies par le professeur CERA et par l'équipe «permanente»). Pendant les 15 premiers jours du programme (première quinzaine de juillet), les conférences et séminaires se succèdent au rythme moyen d'un ou deux par jour. À titre d'illustration, au cours de la session 1993, les sujets suivants ont été traités:

\footnotetext{
- The Question of Descriptive Translation Studies (Dirk Delabastita);

- The Interpreter as a Communication ActorCommunicational, Sociological and Cultural Observations and Question Marks (D. Gile professeur CERA);

- Language and Translation as a Problem in Management (Maddy Janssens);

- How to Study Translational Phenomena (Dirk Delabastita et José Lambert);

- How to Read Publications on Translation (D. Gile);
}

- How to choose a theme for a Research Project (D. Gile);

- Interpretation Research and Theory: Where do We Stand? (D. Gile);

- Advantages and Limitations of Interdisciplinary Research (D. Gile):

- Translation and Mass Communication (Dirk Delabastita et Clem Robyns);

- A Call for Empirical Translation and Interpretation Research (D. Gile);

- Patronage and Translation (André Lefevere);

- Small Empirical Translation and

Interpretation Research Projects (D. Gile);

- Alternatives to Intercultural Borders in

Translation Theory (Anthony Pym);

- Issues in the Assessment of Translation and Interpretation Quality (D. Gile);

- Translation and Business Strategies (José Lambert);

- The Question of Norms (Gideon Toury et Theo Hermans);

- Interpretation and Translation Strategies

(D. Gile);

- The American Connection in Translation Studies (Edwin Gentzler);

- The Translation Politics of the EC (Andreas Poltermann).

Le programme était souple, et permettait d'introduire de nouvelles interventions en cas de nécessité. Notre propre intervention sur le choix des sujets de recherche a été décidée sur place, plusieurs stagiaires ayant déclaré dès les premiers jours du programme qu'ils avaient du mal à se fixer sur un sujet.

La formule actuelle ne cherche pas à assurer l'homogénéité des spécialités des participants. D'ailleurs, lors de la session 1993, les centres d'intérêt annoncés par les stagiaires étaient très variés:

- La traduction catalan-espagnol dans le registre idiomatique;

- L'équivalence dans la traduction;

- Les relations entre la critique de traduction et les études traductologiques descriptives;

- Le sous-titrage:

- Le doublage;

- La méthodologie de l'enseignement de la traduction et de l'interprétation;

- La comparaison des systèmes institutionnels hongrois, allemands et anglais du point de vue de la traduction;

- La politique de la traduction de la poésie contemporaine irlandaise en anglais;

- La condensation du discours en interprétation consécutive;

— La traduction juridique et d'affaires et l'interprétation;

- La communication d'affaires;

- Les proformes anaphoriques dans la simultanée à partir de l'allemand; 
- La traduction de la littérature contemporaine entre l'anglais, le hongrois et le néerlandais;

— La littérature comparée;

— Le discours sur la traduction:

- L'organisation juridique internationale et la communication;

- La communication d'entreprise dans des environnements non occidentaux;

- L'accueil réservé à la littérature occidentale dans les régions sinophones;

- La recherche empirique sur la simultanée dans une optique didactique;

- La traduction d'articles de magazines;

- La sociologie de la traduction.

Nous ne ferons pas une critique précise du contenu et de la structure du programme, qui mériterait une analyse approfondie et une optimisation comme n'importe quel autre programme de formation. Nous nous contenterons de considérer que par nature, il s'agit d'un stage de sensibilisation et d'introduction aux questions méthodologiques fondamentales de la recherche traductologique. Compte tenu de ses aspects particulièrement intéressants tels qu'énumérés plus haut, nous nous interrogerons sur les difficultés potentielles, et partant, sur la faisabilité du projet. On est notamment amené à se poser les questions suivantes :

1. Peut-on répondre, en un mois, aux besoins de stagiaires présentant une si grande variété de centres d'intérêt?

2. Les animateurs peuvent-ils assurer une direction de recherche efficace dans des domaines autres que ceux dont ils sont les spécialistes? Les stagiaires acceptent-ils l'autorité des animateurs qui ne sont pas spécialistes de leur domaine?

3. Les stagiaires acceptent-ils de travailler au sein

d'un groupe aussi varié, où ils sont souvent seuls

à s'intéresser à un thème donné ?

Après notre expérience de la session 1993, les éléments de réponse suivants se dégagent:

Concernant la première question, a priori, le pari semblait risqué: quoi de commun en effet entre la recherche sur l'interprétation de conférence, la recherche sur la traduction d'entreprise et la recherche sur la traduction littéraire? Pendant la première semaine, nos sondages personnels auprès des stagiaires ont révélé une certaine perplexité et quelques réactions négatives face aux interventions ne touchant pas les centres d'intérêt des répondants. Toutefois, par la suite, les attitudes semblent avoir changé, comme il apparaît à travers l'analyse d'un questionnaire rempli à la fin du stage. Ce questionnaire commençait par la question suivante:

How happy are you with the programme? (réponses possibles: Very happy, Happy, Moderately happy, Not too happy, Unhappy)
Sur les 23 stagiaires, 11 ont rempli et rendu le questionnaire. Parmi eux, 9 ont répondu Very happy, et 2 Happy. La satisfaction des stagiaires apparaît donc très clairement. Elle est confirmée par l'unanimité des réponses affurmatives à la question figurant en fin de questionnaire: Would you recommend this programme to other researchers in translation science? Les autres réponses possibles étaient $I$ am not sure, et No. Il va sans dire que le seul indice de satisfaction des stagiaires n'est pas une mesure suffisante de ce qu'ils ont réellement appris. En effet, cette satisfaction peut résulter d'une bonne ambiance et d'une dynamique de groupe, toutes les deux manifestes dans le groupe, ainsi que de l'effet de dissonance, bien connu en psychologie: le fait d'avoir fait l'effort de venir jusqu'à Louvain en renonçant partiellement ou totalement à des congés, éventuellement à un prix financier non négligeable, pourrait prédisposer les stagiaires à se montrer satisfaits, ne serait-ce que pour compenser le «prix» payé.

La réponse à la question passe par d'autres indicateurs, moins facilement accessibles par questionnaire. Le principal est probablement l'évolution constatée dans la manière dont les stagiaires ont posé et analysé leurs problèmes de recherche au fil du programme: en 15 jours, nous avons vu, au cours d'entretiens personnels avec des stagiaires venus nous consulter, plusieurs participants mûrir à partir d'une position souvent naïve et trop générale, pour parvenir à une formulation plus précise et plus critique de leurs sujets. L'une de ces stagiaires, dont nous avions eu l'occasion de commenter un article peu de temps auparavant en soulignant quelques importantes lacunes dans le raisonnement, a écrit un excellent article peu après le stage. Mais un jugement plus précis et fiable ne saurait être fait que dans la durée et dans une optique statistique, sur l'ensemble du groupe, ce qui demandera un certain temps.

Concernant l'aptitude des animateurs à diriger des recherches dans des domaines extérieurs à leur spécialité et l'acceptation, de la part des stagiaires, de l'autorité de ces animateurs, le questionnaire fournit également des éléments de réponse intéressants :

À la question How much did you get out of personal supervision? 7 des répondants ont répondu $A$ lot (le maximum), 5 A fair amount, et aucun n'a répondu Something, Not much ou Very little. Notons qu'un répondant a coché $a$ lot et a fair amount, d'où le total de 12 «notes» et non pas 11 .

Les résultats sont également positifs s'agissant de l'utilité des conférences et "séminaires» des animateurs (par opposition aux conférences du professeur CERA, noté séparément), avec 2 réponses A lot, 8 A fair amount, 1 Something et 1 Not much (1 répondant a indiqué $A$ lot et $N o t$ much, indiquant ainsi la variabilité de l'intérêt de ces séminaires et conférences pour lui).

Des réponses au questionnaire, il ressort aussi que les entrevues personnelles avec les instructeurs ont été jugées plus utiles que les séminaires et conférences de ces demiers. La raison en est probablement 
la possibilité qui était donnée aux participants, lors des rencontres personnelles, de poser aux animateurs des questions qui les concernaient directement. En tout état de cause, les stagiaires semblent avoir accepté l'autorité de ces derniers en matière de recherche. La chose est très encourageante quand on s'interroge sur les possibilités de coopération interdisciplinaire. On peut toutefois penser que les résultats pourraient être moins positifs pour des stagiaires plus avancés dans leur recherche, en ce sens que leurs préoccupations pourraient être plus techniques et appeler des compétences plus spécialisées que n'auraient pas des directeurs de recherche étrangers au domaine concerné.

Autres éléments qui incitent à l'optimisme concernant l'apport possible d'une équipe d'animateurs interdisciplinaire, les réponses à deux autres questions:

La première: How much did you get out of the programme that is directly relevant to your personal project?

Sur les 11 répondants, 5 ont répondu $A$ lot, $5 \mathrm{~A}$ fair amount, et $1 \mathrm{I}$ got something out of the programme; personne n'a répondu Not much ou Very little. Il s'avère donc qu'en dépit de la grande diversité des centres d'intérêt et projets de recherche des stagiaires, le programme leur a apporté des éléments qu'ils considèrent comme directement utiles. Là aussi, on est fondé à penser que les résultats auraient pu être moins positifs si les stagiaires avaient été plus avancés dans leur recherche.

Dans le même esprit, à la question de savoir si pour les interprètes, les discussions sur la traduction, et pour les traducteurs, les discussions sur l'interprétation ont été utiles, 1 répondant les a jugées très utiles, 6 utiles, 2 moyennement utiles, 1 utiles ou moyennement utiles, et 1 pas très utiles.

Enfin, les stagiaires semblent avoir beaucoup apprécié le contact avec les autres stagiaires. À la question de savoir How much did you get out of the contact with other participants? 7 ont répondu $a$ lot et 4 a fair amount. Une dynamique de groupe semble donc s'être instaurée, avec un intérêt réciproque, de profondeur variable, pour les travaux des uns et des autres. En conclusion, la diversité des centres d'intérêt, qui était pour nous la principale source d'inquiétude, ne semble donc pas avoir posé de véritable problème. Nous avons d'ailleurs constaté un phénomène analogue dans un cours de méthodes de recherche en études japonaises que nous donnons à l'intention des étudiants de maîtrise au Département Corée-Japon de 1'Institut National des Langues et Civilisations Orientales de Paris. Là aussi, les étudiants ont des centres d'intérêt variés, qui vont de la littérature à l'histoire en passant par l'économie, la linguistique et la sociologie et l'art, mais en cours d'année, ils semblent apprendre à s'intéresser aux travaux qui ne les concernent pas directement en raison de leur apport méthodologique. Nous pensons toutefois que le phénomène s'applique essentiellement aux débutants. S'agissant de , chercheurs con- firmés, les problèmes méthodologiques deviennent probablement en partie plus spécifiques, et la partie d'intérêt commun rétrécit d'autant.

Cette observation nous conduit à un autre commentaire: en l'état actuel, il semble que la formule CERA ne soit pas en mesure d'assurer l'homogénéité du niveau des participants en matière de connaissances et d'expérience de la recherche, car le recrutement des stagiaires est quelque peu aléatoire, avec quelques décisions tardives, voire de demière minute. Il en résulte la possibilité de cristallisation sur place d'un groupe mixte, des débutants côtoyant des chercheurs confirmés, ce qui ne saurait manquer d'engendrer des problèmes. En l'occurrence, pour la session 1993, le niveau était homogène, la quasitotalité des stagiaires étant débutants. Il nous semble intéressant, dans l'éventualité de la constitution d'autres programmes analogues à celui de la formule CERA, de bien s'assurer de l'homogénéité du groupe en définissant très clairement, dans les documents d'information annonçant et expliquant le programme, le niveau de connaissances et d'expérience visé.

En rapport avec cette observation, si l'apport des animateurs ayant des antécédents littéraires s'est avéré considérable, c'est à notre avis en raison de leur ouverture d'esprit et de leur capacité (et leur volonté) d'appliquer leurs raisonnements à d'autres sujets que ceux qu'ils connaissaient bien. Là aussi, face à des chercheurs confirmés pour qui les problèmes auraient été plus complexes et plus spécifiques, la formule aurait probablement perdu de sa puissance.

En tant que partie prenante, il nous est difficile de porter un jugement objectif sur le contenu de la session 1993. Il nous semble cependant important de souligner la serviabilité et la générosité en temps, en documentation et en efforts de toutes sortes, de toute l'équipe des permanents à l'égard des stagiaires (et du professeur CERA). Une telle attitude chez une ou deux personnes aurait pu être le reflet de leur personnalité individuelle; généralisée à toute l'équipe, elle nous semble relever d'un esprit CERA, probablement attribuable à son fondateur et animateur principal. Il est fort possible que cette ambiance, plus amicale que formelle, ait joué un rôle très important dans la réussite du programme, étant donné la petite taille du groupe et l'intensité du travail.

En conclusion, d'après les réactions recueillies à la fin du programme 1993, nous pensons que pour les débutants, celui-ci a été très bénéfique: ouverture d'horizons nouveaux en matière d'approches et de raisonnements traductologiques, ouverture psychologique à des centres d'intérêts étrangers aux préccupations individuelles de chaque participant, orientation par des chercheurs confirmés, possibilité de présenter en public des résultats de recherche, contacts avec d'autres chercheurs débutants, enrichissement bibliographique, accès personnel et direct au monde de la recherche par le biais de chercheurs confirmés, contacts personnels avec ces chercheurs. Aucun autre programme n'offre, à notre connaissance, un tel apport à des débutants dans la recherche 
traductologique. Notons aussi que les sessions des années précédentes ont apparemment permis à de jeunes chercheurs de faire leur entrée dans le microcosme des chercheurs traductologues, avec des articles, des communications et des participations à différents colloques et congrès. En revanche, le programme n'a pas abordé des techniques précises telles que les méthodes de sondage, d'échantillonnage, de mesure, d'inférences statistiques. Nous pensons que de telles techniques ne sauraient pour l'instant être apprises ailleurs que dans des cours de méthodologie dans des disciplines bien établies dans la tradition de la recherche empirique (psychologie, éducation, sociologie, médecine, etc.).

Pour des chercheurs plus avancés, qui auront déjà la base méthodologique, la formule nous semble devoir être quelque peu adaptée, avec, comme il est dit plus haut, une sélection de candidats ayant un niveau plus ou moins homogène et des centres d'intérêt proches. Il faudrait aussi dans un tel cas recruter des intervenants susceptibles d'apporter une grande valeur ajoutée en techniques et en connaissances précises, dont des spécialistes des domaines concernés. A priori, la configuration à Louvain est plutôt favorable au lancement des débutants qu'à ce deuxième cas de figure.

La formule CERA nous semble exemplaire à plus d'un titre étant donné la nature et l'état de la traductologie dans le monde. Telle quelle (mais avec des aménagements nécessaires à son optimisation dans chaque environnement spécifique), elle pourrait être reproduite ailleurs, éventuellement au Canada, en Amérique latine, en Asie, en Australie, là où les traductologues compétents sont dispersés et où il y a une demande de la part de jeunes chercheurs. Adaptée, elle pourrait être reprise pour des stages spécialisés, de 15 jours par exemple, dans des domaines spécifiques pour chercheurs confirmés, et remplacer avantageusement certains colloques et conférences, qui sont en passe de devenir trop nombreux, et donc répétitifs.

DANIEL GILE

INALCO, Paris et Université Lumière, Lyon, France

\section{RÉFÉRENCES}

DELABASTITA, Dirk (1992) : «How to Study Translation? Experiences from the CERA Workshops», article intégré dans le 'kit' de présentation du programme CERA pour 1992.

GILE, Daniel (1991) : «Methodological Aspects of Interpretation (and Translation Research)», Target, 3/2, pp. 153-174.

HOLMES, James S. (1988) (1972, 1975) : «The Name and Nature of Translation Studies», James S. Holmes (Ed), Translated Papers on Literary Translation and Translation Studies, Amsterdam, Rodopi (Approaches to Translation Studies 7).

LAMBERT, José et Hendrik VAN GORP (1985) : «On Describing Translations», Theo Hermans
(Ed), The Manipulation of Literature Studies in Literary Translation, London, Croom Helm.

TOURY, Gideon (1980) : In search of a Theory of Translation, Tel-Aviv, the Porter Institute.

TOURY, Gideon (1991) : «Experimentation in Translation Studies: Achievements, Prospects and some Pitfalls», Sonja Tirkkonen-Condit (Ed), Empirical Research in Translation and Cultural Studies: Selected Papers of the TRANSIF Seminar, Savonlinna 1988, Tübingen, pp. 45-66. 